\title{
The 46th annual meeting of the european society for blood and marrow transplantation: reviewers
}

C Springer Nature Limited 2020

29 August-1 September, 2020 Virtual Meeting

Modified and published with permission from https://www.ebmt.org/annual-meeting

Sponsorship Statement: Publication of this supplement is sponsored by the European Society for Blood and Marrow Transplantation.

We would like to thank the following reviewers for their generous support (2019)

\section{Physicians:}

Manuel Abecasis, Michael Albert, Tobias Alexander, Persis Amrolia, Marc Ansari, Jane Apperley, Mutlu Arat, Renate Arnold, Holger Auner, Diana Averbuch, Franco Aversa, Francis Ayuk, Ulrike Bacher, Andrea Bacigalupo, Peter Bader, Claudia Baldus, Adriana Balduzzi, Frederic Baron, John Barrett, Grzegorz Basak, Jacques-Olivier Bay, Ali Bazarbachi, Dietrich Beelen, Andreas Beilhack, Meral Beksac, Arantxa Bermudez, Maria Ester Bernardo, Alice Bertaina, Wolfgang Bethge, Joan Bladé, Didier Blaise, Jaap Jan Boelens, Francesca Bonifazi, Halvard Bönig, Chiara Bonini, Claire Booth, Martin Bornhäuser, Aida Botelho de Sousa, Robbert Bredius, Marco Bregni, Eolia Brissot, Peter Brossart, Mats Brune, Gesine Bug, Andreas Burchert, Boris Calmels, Fernando Campilho, António Campos, Jonathan Canaani, Enric Carreras, Luca Castagna, Toni Cathomen, Simone Cesaro, Christian Chabannon, Yves Chalandon, James Chim, Fabio Ciceri, Selim Corbacioglu, Catherine Cordonnier, Jan Cornelissen, Fernando Costa, Charles Craddock, Krzysztof Czyzewski, Maud D'Aveni, JeanHugues Dalle, Ugo De Giorgi, Rafael de la Cámara, Josu de la Fuente, John de Vos, Theo De Witte, Raynier Devillier, Raynier Devillier, Tamara Diesch, Sascha Dietrich, Peter Dreger, Carlo Dufour, Remy Dulery, Jean El-Cheikh, Jordi Esteve, Mathias Eyrich, Fred Falkenburg, Catherine Faucher, Lawrence Faulkner, Boris Fehse, Roland Fenk, Isabelina Ferreira, Tobias Feuchtinger, Jürgen Finke, Katharina Fleishhauer, Yngvar Fløisand, Jürgen Föll, Edouard Forcade, Laurent Garderet, Jordan Gauthier, Andy Gennery, Catarina Geraldes, Brenda Gibson, Sebastian
Giebel, Lidia Gil, Maria Gilleece, Christian Gisselbrecht, Marta González Vicent, Norbert-Claude Gorin, Martin Gramatzki, Raffaella Greco, Hildegard Greinix, Bernd Gruhn, José Guimarães, Tayfun Güngör, Joerg Halter, Amir Ali Hamidieh, Rupert Handgretinger, Shahrukh Hashmi, Wolfgang Herr, Aloysius Ho, Ernst Holler, Katharine Hsu, Kai Hübel, Michael Hudecek, Cecilie Hveding Blimark, Elad Jacoby, Madan Jagasia, Gregorio Jaimovich, Krzysztof Kalwak, Majid Kazmi, Mohamed Kharfan-Dabaja, Kirill Kirgizov, Katharina Kleinschmidt, Thomas Klingebiel, Guido Kobbe, Ulrike Koehl, Christian Koenecke, Angela Krackhardt, Nicolaus Kroeger, Jurgen Kuball, Austin Kulasekararaj, Meltem Kurt, Chara Kyriakou, João Lacerda, Peter Lang, Arjan Lankester, Francesco Lanza, Jérôme Larghero, Anita Lawitschka, Matthieu Leclerc, Steven LeGouill, Claudia Lengerke, Per Ljungman, Juan Carlos Llamas, Franco Locatelli, Sonja Loges, Mark Lowdell, Thomas Luft, Andreas Mackensen, Florent Malard, David Marks, Judith Marsh, Susanne Matthes-Leodolter, Donal McLornan, Roland Meisel, Isabelle Meyts, Mauricette Michallet, Gérard Michel, David Michonneau, Stephan Mielke, Malgorzata Mikulska, Noel Milpied, Nuno Miranda, Mohamad Mohty, Silvia Montoto, John Moore, Emma Morris, Ingo Müller, Paolo Muraro, Arnon Nagler, Andrea Necchi, Bénédicte Neven, Dietger Niederwieser, Charlotte Niemeyer, Shinishiro Okamoto, Eduardo Olavarria, Attilio Olivieri, Fabrizio Pane, jakob Passweg, Steven Pavletic, Paolo Pedrazzoli, Régis Peffault de la Tour, Olaf Penack, Zinaida Peric, Christina Peters, Kavita Raj, Alessandro Rambaldi, Carlos Ramos, Antonio Risitano, Marie Robin, Stephen Robinson, Martino Rodrigo, Montserrat Rovira, Marie-Thérèse Rubio, Annalisa Ruggeri, Tapani Ruutu, Riccardo Saccardi, Nina Salooja, Sujith 
Samarasinghe, Jaime Sanz, Martin Sauer, Bipin Savani, Johannes Schetelig, Paul Schlegel, Patrick Schlegel, Christoph Schmid, Martin Schmidt-Hieber, Helene Schoemans, Stefan Schoenland, Harry Schouten, Hubert Schrezenmeier, Petr Sedlacek, Markus Seidel, Bronwen Shaw, Avichai Shimoni, Roni Shouval, Mary Slatter, Emilian Snarski, John Snowden, Gerard Socie, Carlos Solano, Carsten Speckmann, Alexandros Spyridonidis, Micha Srour, Matthias Stelljes, Brigitte Strahm, Jan Styczynski, Anna Sureda, Louis Terriou, Antoine Toubert, Olivier Tournilhac, Fernanda Trigo, Anja Troeger, Evelyn Ullrich, Alvaro Urbano-Ispizua, Luca Vago, Michel Van Gelder, Elizabeth Vandenberghe, Paul Veys, Reinhard Voll, Lotte Wietten, Andre Willasch, Thomas Wölfel, Daniel Wolff, Nina Worel, Depei Wu, Gerald Wulf, Ibrahim YakoubAgha, Akif Yesilipek, Robert Zeiser

\section{Nurses Group:}

Erik Aerts, Iris Agreiter, Janet Baker, Caroline Bompoint, Diana Comerford, Maaike de Ruijter, Ariadna Domenec, Rose Ellard, Seckin Erdal, Letizia Galgano, Marjola Gjergji, Kathy Goris, Martine Heylens, Daphna Hutt, Helen Jessop, Klara Kalatovak, Birgit Keinrath, Michelle Kenyon, Annika Kisch, Polona Klanšček, Sarah Liptrott, Anna Mantecon, Francisco Marquez, Hilda Mekelenkamp, John Murray, Iwona Przewozna, Marijke Quaghebeur, Julia Ruiz
Pato, Isabel Salcedo, Sandra Schoenfeld, Marieke Schoordijk, Teija Schröder, Simon Slade, Sarah Sprenger, Orla Stewart, Jacqui Stringer, Eugenia Trigoso, Marie Waller, Elisabeth Wallhult

\section{Psychiatry and Psychology Group:}

Anna Barata, Angela Scherwath, Frank SchulzKindermann

\section{Pharmacist Committee:}

Rick Admiraal, Tiene Bauters, Claudia Langebrake, Karen Vermis

\section{Data Management Group:}

Myriam Labopin, Anne Lippinkhof, Emmanuelle Polge, Annelot van Amerongen, Silvia Zaccagnino

\section{Quality Management Group:}

Deborah Anderson, Julie Dolva, Songül Durmaz, Anne Emmett, Simon Hack, Phuong Huynh, İlknur Kozanoğlu, Olga Lopez, Mara Magri, Renza Monteleone, Tuula Rintala, Nick van Sinderen

\section{Statistics Group:}

Ariane Boumendil, Jacques-Emmanuel Gallimard, Myriam Labopin, Giulia Sbianchi 